\title{
Magnetic Archimedean Tessellations in Metal-Organic Frameworks
}

\author{
Hua Chen, Laura Voigt, Mariusz Kubus, Dmytro Mihrin, Susanne Mossin, René Wugt Larsen, Søren Kegnces, \\ Stergios Piligkos and Kasper S. Pedersen*
}

The self-assembly of trivalent lanthanide ions with ditopic organic spacers results in the formation of complex tiling patterns that mimic the structural motifs of quasi-periodic $2 D$ materials. The assembly of trans- $\left\{L_{n} I_{2}\right\}^{+}$nodes $(\mathrm{Ln}=\mathrm{Gd}$, Dy) with both closed-shell and anion radicals of 4,4'bipyridine affords rare examples of Archimedean tessellations in a metal-organic framework. We furthermore demonstrate occurrence of sizable magnetic exchange interactions and slow relaxation of magnetization behaviour in a complex tessellation pattern. The implementation of Archimedean tessellations in lanthanide(III) coordination solids couriers a strategy to design elusive quasi-periodic metal-organic frameworks with inimitable magnetic properties.

The design and realization of complex and aperiodic twodimensional tessellations in molecule-based materials constitutes a novel route to harvest physical properties, for instance photonic, electronic, magnetic, and phononic characteristics, which are expected to be unparalleled compared to their periodic counterparts. ${ }^{[1-3]}$ However, applications are elusive due to the severe scarcity of materials exhibiting the desired structural motifs. The two-dimensional dodecagonal quasicrystalline phase (Fig. 1a, ddQC) is well-known in both hard and soft materials, as well as in supramolecular networks. ${ }^{[4}$ ${ }^{6]}$ Herein, the tessellation of triangles and squares, present in a ratio of $4 / \sqrt{ } 3 \approx 2.3$, leads to the disappearance of periodicity and the formation of local 12-fold rotational symmetry. Generally, quasicrystals are found in the vicinity of structurally related, periodic structures and the ddQCs, specifically, often co-occur with the periodic Archimedean tessellations (ATs, Fig. 1bc), which are therefore referred to as quasicrystal approximants. ${ }^{[7]}$ Interestingly, the sole example of a quasicrystal phase found in a metal-organic network structure was realized in single-atom layers, by co-evaporating large Eu atoms and organic linkers. ${ }^{[8-}$ ${ }^{10]}$ The construction of five- and six-fold nodes is critical for the realization of such structures and necessitates confining five or six chemical bonds to the plane (cf. Fig. 1a). This requirement precludes the use of most transition metal-ions as nodes but may be met by larger metal ions as those found in the f-block. In the fragile single-layer materials, the exact chemical nature, such as

[*] H. Chen, Dr. L. Voigt, Dr. Mariusz Kubus, Dr. D. Mihrin, Prof. Dr. S. Mossin, Prof. Dr. R. W. Larsen, Prof. Dr. S. Kegnæs, Prof. Dr. K. S. Pedersen

Department of Chemistry

Technical University of Denmark

Kemitorvet, Building 207, DK-2800 Kgs. Lyngby, Denmark

E-mail: kastp@kemi.dtu.dk (K.S.P.)

Web: www.kemi.dtu.dk/pedersen

Prof. Dr. S. Piligkos

Department of Chemistry

University of Copenhagen

DK-2100 Copenhagen, Denmark

Supporting information and the ORCID identification number(s) for the author(s) of this article can be found under:

https://doi.org/10.1002/ the oxidation state of the $4 \mathrm{f}$ ion nodes, remains unknown. In the more robust, bulk coordination networks and metal-organic frameworks (MOFs), quasicrystals are elusive. ${ }^{[1]}$ This may partly be related to the complications in their crystallographic identification and partly due to the scarcity of structures that bear the potential to express quasicrystallinity. The elongated triangular $\left(3^{3} .4^{2}\right)$ and snub square $\left(3^{2} .4 .3 .4\right)$ ATs (Fig. 1bc) each possess a triangle-to-square ratio of 2 and have been desired structural motifs in the quest for ddQCs. However, even realizations of MOF structures complying to these ATs are exceedingly scarce. a

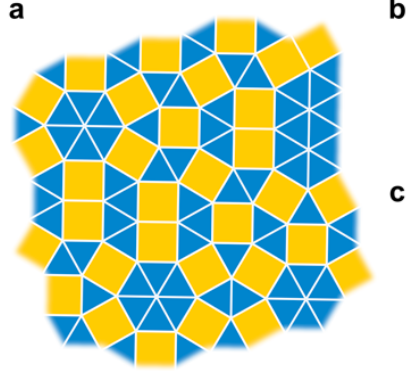

b
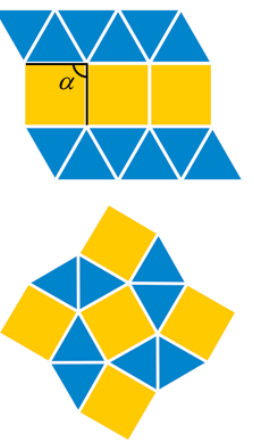

Figure 1. (a) An example of a random-tiling ddQC, (b) the semiregular elongated triangular tiling, and (c) the semiregular snub square tiling.

Taking advantage of the common pentagonal bipyramidal coordination motif of the uranyl ion, trans- $\left\{\mathrm{UO}_{2}\right\}^{2+}$, Smetana et al. reported the first example of a snub square tiling in a metalorganic bulk material. ${ }^{[1]]}$ We recently reported the utilization of trans- $\left\{\mathrm{YbI}_{2}\right\}$ nodes as five-vertex building units to form the triangular elongated tiling in $\mathrm{YbI}_{2}$ (bipy) $)_{5 / 2}$ (bipy $=4,4^{\prime}$ bipyridine; cf. Fig. 1b). ${ }^{[12]}$ The lanthanide ions are particularly relevant for the realization of both ATs and ddQC phases as the coordinative plasticity allows for their incorporation in a variety of local coordination geometries. Furthermore, their weak interactions with ligands are commensurable with the weak interactions typically intrinsic to aperiodic structures. ${ }^{[1]}$ The construction of the $\mathrm{YbI}_{2}$ (bipy) $)_{5 / 2}$ framework necessitated the introduction of divalent lanthanide units, whose preponderance in chemistry is dwarfed by the trivalent ones. To circumvent this problem, we herein demonstrate a novel strategy to design molecule-based magnetic Archimedean tessellations incorporating trivalent lanthanide ions.

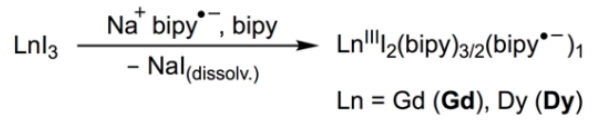

Scheme 1. Synthetic route to $\mathrm{Gd}$ and Dy.

The reaction of sodium metal with bipy was early reported to generate the anion radical bipy ${ }^{\bullet} .{ }^{[13]}$ However, to date, only a 
single crystalline compound is known to incorporate this chemical species, $\mathrm{Na}^{+}(\mathrm{en})\left(\right.$ bipy $\left.^{\circ}\right)$ (en = ethane-1,2-diamine).$^{[14]}$ The reaction of sodium with an excess of bipy in $\mathrm{CH}_{3} \mathrm{CN}$ affords an intensely dark-blue colored solution. The X-band electron paramagnetic resonance (EPR) spectrum of the frozen solution yields a single narrow resonance at $g=2.00$ (Fig. S1, Supporting Information), compatible with the formation of an organic radical and similar to that of $\mathrm{Na}^{+}$(en)(bipy $\left.{ }^{\circ}\right)(g=2.00429){ }^{[14]}$ The addition of solid $\mathrm{GdI}_{3}$ to the bipy ${ }^{\circ} /$ bipy solution results in an immediate formation of a dark-blue microcrystalline powder which exhibits a broad EPR signal (Fig. S2, Supporting Information). The elemental analyses of $\mathrm{C}, \mathrm{H}, \mathrm{N}, \mathrm{Gd}, \mathrm{I}$, and $\mathrm{Na}$ indicate a formulation of $\mathrm{GdI}_{2}$ (bipy) $)_{5 / 2} \cdot \mathrm{CH}_{3} \mathrm{CN}$ and no presence of $\mathrm{Na}^{+}$(cf. Supporting Information). Careful layering of the darkblue bipy ${ }^{\bullet} /$ bipy solution onto $\mathrm{CH}_{3} \mathrm{CN}$-covered solid $\mathrm{GdI}_{3}$ affords block-like, dark-blue crystals of $\mathbf{G d}$ suitable for singlecrystal X-ray diffraction. Gd crystallizes in the tetragonal $I 4_{1} 22$ space group and features an ideal snub square tessellation of $\mathrm{GdI}_{2}$ (bipy) $)_{5 / 2} \cdot x \mathrm{CH}_{3} \mathrm{CN}$ (cf. Fig. 2a).

a

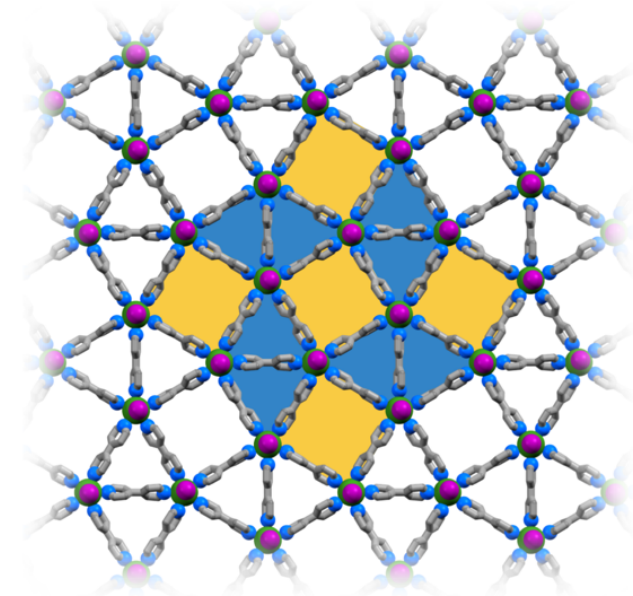

b

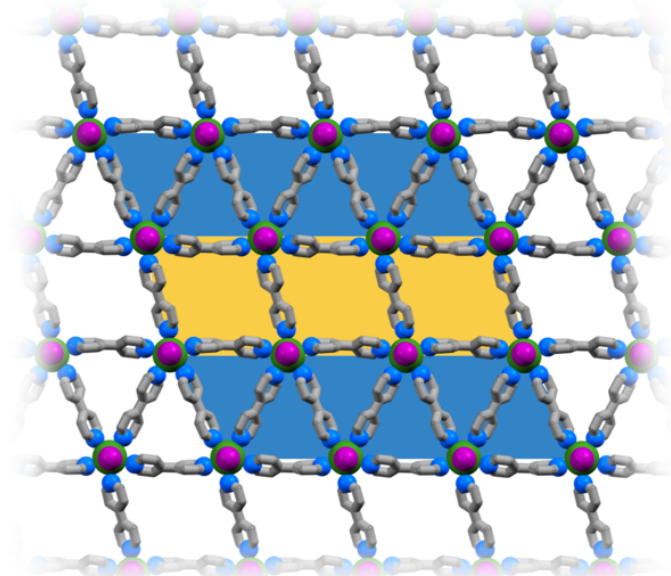

Figure 2. (a) Single-crystal X-ray structure of Gd viewed perpendicular to one of the snub square tiling layers. (b) Single-crystal structure of the trace impurity phase Gd'. Color codes: Gd, turquoise; I, purple; N, blue; C, grey. $\mathrm{H}$ atoms and co-crystallized $\mathrm{CH}_{3} \mathrm{CN}$ molecules have been omitted for clarity.
The coordination geometries of the two crystallographically independent $\mathrm{Gd}$ centers are almost identical. The Gd-I bond lengths of 3.0437(7)-3.054(2) $\AA$ are slightly longer than those found in trans $-\left[\mathrm{Gd}^{\mathrm{III}} \mathrm{I}_{2}(\text { thf })_{5}\right]^{+}$(thf $=$tetrahydrofuran) of 3.00 $\AA,{ }^{[15]}$ albeit significantly shorter than the Eu- I bonds found in trans-[ $\left[\mathrm{Eu}^{\mathrm{II}} \mathrm{I}_{2}(\mathrm{thf})_{5}\right]$ of 3.22-3.24 $\AA,{ }^{[16]}$ corroborating the presence of $\mathrm{Gd}(\mathrm{III})$, and not the extremely rare $\mathrm{Gd}(\mathrm{II})$, in $\mathbf{G d}{ }^{[17]}$ The $\mathrm{N}-\mathrm{Gd}-\mathrm{N}$ angles are in the range of $68.1(2)^{\circ}$ to $75.3(3)^{\circ}$ and together with the $\mathrm{I}-\mathrm{Gd}-\mathrm{I}$ linearity $\left(179.23(4)^{\circ}, 177.90(5)^{\circ}\right)$ reflect the close proximity of the local coordination environment to $D_{5 \mathrm{~h}}$ symmetry. The presence of Gd(III) necessitates one bipy ${ }^{\bullet}$ radical ligand per $\mathrm{GdI}_{2}$ (bipy) 5/2 $_{\text {f }}$ formula unit. Goicoechea and coworkers have previously shown that the inter-pyridinic bond in bipy shortens by $\sim 4 \%$ upon one-electron reduction. ${ }^{[14]}$ According to that, in Gd, the two crystallographically independent $\mathrm{Gd}$ centres are each surrounded by three bipy ${ }^{0 / \bullet}$ ligands with longer $\mathrm{C}-\mathrm{C}$ bond lengths of 1.49(3)-1.51(2) $\AA$ and two bipy ${ }^{0 / \bullet-}$ ligands with short $\mathrm{C}-\mathrm{C}$ bond lengths of $1.45(2) \AA$ corresponding to a reduction of $\sim 4 \%$. Interestingly, the anticipated localized bipy ${ }^{\circ}$ ligands span the edges of the triangles in only one direction of the plane which leads to the formation of $\left\{\mathrm{Gd}_{4}{ }_{4}{ }_{4}\left(\text { bipy }^{\bullet}\right)_{4}\right\}$ rhombi (Fig. S3). The presence of mixed valency in the bipy ${ }^{0 / \bullet}$ scaffold could be expected to lead to strong inter-valence charge transfer (IVCT) transitions in the mid- or near-infrared spectrum as e.g. observed in transition metal complexes of mixed-valent 2,2'-bipyridine ${ }^{0 / \bullet} \cdot{ }^{[18]}$ However, no such IVCT bands could be observed in Gd (Fig. S6, Supporting Information) which may, tentatively, be attributed to the weakly covalent nature of metal-ligand bonds and the localization of the unpaired electron, as inferred from crystallography. Notably, in each crystallization vial, a few needle-shaped dark-blue crystals of $\mathbf{G d}^{\prime}$ were systematically obtained. The structural analysis of $\mathbf{G d}^{\prime}$ revealed an identical chemical composition of $\mathrm{GdI}_{2}$ (bipy) $)_{5 / 2} \cdot x \mathrm{CH}_{3} \mathrm{CN}$, but $\mathbf{G d}^{\prime}$ crystallizes in the triclinic $P \overline{1}$ space group and resembles an elongated triangular tiling similar to the previously reported $\mathrm{YbI}_{2}$ (bipy) $)_{5 / 2}$ structure (Fig. 2b). However, in contrary to $\mathrm{YbI}_{2}$ (bipy) $)_{5 / 2}$, the tilting angle, $\alpha$ (cf. Fig. 1b), departs significantly from $90^{\circ}$ and amounts to $101^{\circ}$. Thus, the Gd centres could, as well, be considered as approaching defect 6-fold nodes, corresponding to $\alpha=120^{\circ}$. Similarly to Gd, two fifths of the bipy display short inter-pyridinic $\mathrm{C}-\mathrm{C}$ bonds of an average length of $1.44(2) \AA$ and three fifths exhibit normal, longer $\mathrm{C}-\mathrm{C}$ bonds of 1.51(1)-1.53(2) $\AA$, echoing the existence of Gd(III) and both bipy and bipy ${ }^{\bullet}$ in $\mathbf{G d}^{\prime}$. Use of $\mathrm{DyI}_{3}$ reveals an identical behavior and yields DyI $_{2}$ (bipy) $)_{5 / 2} \cdot x \mathrm{CH}_{3} \mathrm{CN}$ (Dy). Dy is isostructural to Gd with slightly contracted bond lengths. Notably, no traces of an elongated triangular tessellation phase could be observed for Dy.

The room temperature value of the magnetic susceptibilitytemperature product, $\chi T$, of $\mathbf{G d}$ amounts to $8.6 \mathrm{~cm}^{3} \mathrm{~K} \mathrm{~mol}^{-1}$, close to the value expected for an uncorrelated pair of a $\mathrm{Gd}(\mathrm{III})$ ion $\left(4 \mathrm{f}^{7}\right)$ and an organic radical $(S=1 / 2, g=2.0)$ of $8.3 \mathrm{~cm}^{3} \mathrm{~K}$ $\mathrm{mol}^{-1}$. The $\chi T$ product decreases only slightly by cooling reflecting the relatively weak $\mathrm{Gd}^{\mathrm{III}}$-bipy ${ }^{\circ}$ superexchange interactions. Similarly, the field-dependence of the magnetization, $M$ vs $H$, reveals that $M$ saturates at low temperature at $8.0 \mu_{\mathrm{B}}$, as expected for one $\mathrm{Gd}^{\mathrm{III}}$ and one bipy ${ }^{\bullet}$ per formula unit, only weakly magnetically coupled. The structural analyses suggested the presence of $\left\{\mathrm{Gd}^{\mathrm{III}}{ }_{4}\left(\text { bipy }^{\bullet}\right)_{4}\right\}$ 


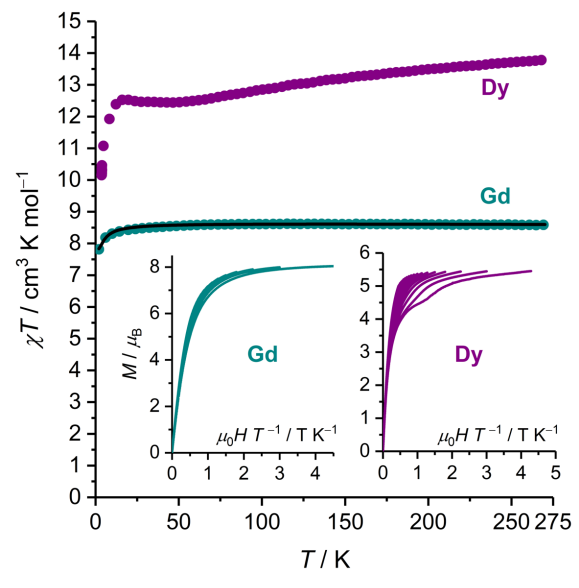

Figure 3. Temperature dependence of the magnetic susceptibility-temperature product, $\chi T$, for polycrystalline $\mathbf{G d}$ and $\mathbf{D y}$ obtained under a dc magnetic field of $\mu_{0} H=1000$ Oe. The solid black line is the best fit as described in the main text. The insets show the magnetic-field dependence of the magnetization, $M$, plotted against $\mu_{0} H T^{-1}$.

rhombi. Herein, the adjacent $\mathrm{Gd}^{\mathrm{III}}$ and bipy ${ }^{\bullet}$ are expected to be coupled by the superexchange mechanism. Thus, the magnetization data were modelled using a spin-Hamiltonian (Eq. 1) for an eight-membered ring of alternating $\mathrm{Gd}(\mathrm{III})$ ions and bipy ${ }^{\bullet}$ radicals:

$$
\begin{aligned}
\widehat{\mathcal{H}}= & g \mu_{\mathrm{B}} \mu_{0} H \sum_{i} \hat{S}_{i}+J\left(\hat{S}_{\mathrm{Gd} 1} \hat{S}_{\mathrm{rad} 1}+\hat{S}_{\mathrm{Gd} 2} \hat{S}_{\mathrm{rad} 1}+\hat{S}_{\mathrm{Gd} 2} \hat{S}_{\mathrm{rad} 2}+\hat{S}_{\mathrm{Gd} 3} \hat{S}_{\mathrm{rad} 2}\right. \\
& \left.+\hat{S}_{\mathrm{Gd} 3} \hat{S}_{\mathrm{rad} 3}+\hat{S}_{\mathrm{Gd} 4} \hat{S}_{\mathrm{rad} 3}+\hat{S}_{\mathrm{Gd} 4} \hat{S}_{\mathrm{rad} 4}+\hat{S}_{\mathrm{Gd} 1} \hat{S}_{\mathrm{rad} 4}\right)
\end{aligned}
$$

where $g=2$ is the isotropic $g$ factor for both $\mathrm{Gd}^{\mathrm{III}}$ and the radical spins, $\mu_{\mathrm{B}}$ is the Bohr magneton, $H$ is the magnetic field, $\hat{S}$ is a spin operator indexed appropriately for each $\mathrm{Gd}^{\mathrm{III}}$ and bipy ${ }^{\circ}$, and $J$ is the coupling constant. The first term represents the Zeeman interaction for all $\mathrm{Gd}^{\mathrm{III}}$ and radical spins and the second term accounts for the superexchange interaction. Anisotropy terms are neglected since they are expected to be small for $\mathrm{Gd}^{\mathrm{III}}$ and for the bipy ${ }^{\bullet}$ radical. The dimension of the matrix representation of Eq. 1 for $\mathbf{G d}$ is 65536, which is impractical with respect to standard numerical full matric diagonalization approaches. Thus, for the quantitative interpretation of the magnetic properties of $\mathbf{G d}$, we used home-written software (ITO-MAGFIT) ${ }^{[19]}$ that makes use of irreducible tensor operator algebra ${ }^{[20]}$ to block-diagonalize the spin-Hamiltonian and the Levenberg-Marquardt algorithm ${ }^{[21]}$ to fit the magnetization data. The $\chi T$ product and the $M$ vs $H$ data of Gd were simultaneously fitted to spin-Hamiltonian (Eq. 1). This resulted to the best fit superexchange coupling constant $J / h c$ $=0.073(4) \mathrm{cm}^{-1}$ (Fig. 3, black trace, and Fig. S7, Supporting Information). Under these conditions, the spin ground state of Gd is an $S=12$ (Fig. S8, Supporting Information), separated from the first excited state, a degenerate doublet of $S=11$ states, by only $0.16 \mathrm{~cm}^{-1}$ at zero magnetic field. This vanishing value of $J$ is noteworthy although not uncommon for $\mathrm{Gd}(\mathrm{III})$-radical complexes. ${ }^{[22]}$ For $\mathbf{D y}$, the room temperature $\chi T$ product leans at $13.8 \mathrm{~cm}^{3} \mathrm{~K} \mathrm{~mol}^{-1}$, which is only slightly lower than the expected value for a $\mathrm{Dy}^{3+}$ ion with a ${ }^{8} \mathrm{H}_{15 / 2}$ ground term $\left(C=14.2 \mathrm{~cm}^{3} \mathrm{~K}\right.$ $\mathrm{mol}^{-1}$ ) and a bipy ${ }^{\bullet}$ ligand. The $\chi T$ product decreases slightly at

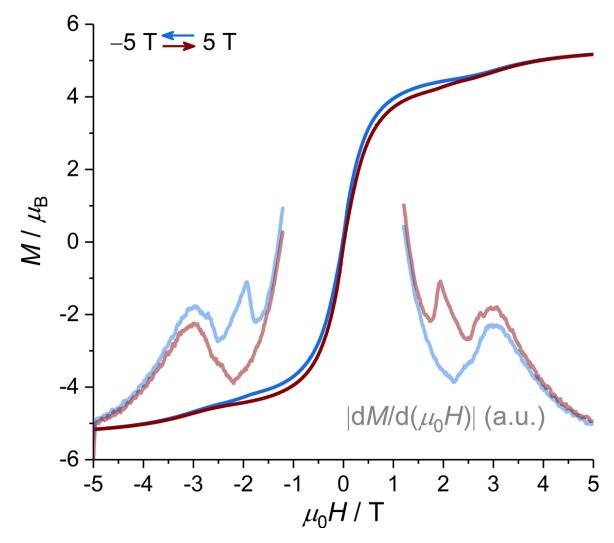

Figure 4. $\mathrm{M}$ vs $\mu_{0} \mathrm{H}$ data obtained at $T=2.1 \mathrm{~K}$ (sweeping rate of $200 \mathrm{Oe} \mathrm{s}^{-1}$ ) and the first derivative, $\mathrm{d} M / \mathrm{d}\left(\mu_{0} H\right)$.

lower temperatures due to depopulation of excited ligand field states. Below $c a .15 \mathrm{~K}$, the $\chi T$ product drops abruptly, which may be ascribed to magnetic superexchange interactions. The $M$ vs $H$ data (Fig. 3 (inset), Fig. 4) reveal a clear step at $c a .3 \mathrm{~T}$. Notably, a similar step feature was previously observed in a Dy(III)-radical chain system, but absent in the isostructural Gd(III) system, and attributed to the presence of significant exchange interactions in the Dy(III) system. ${ }^{[23,24]}$ At fields smaller than $\sim 3 \mathrm{~T}$, the $M$ vs $H$ data show the opening of a small hysteresis gap that collapses again when zero field is approached (Fig. 4). Several $D^{3+}$ complexes with approximate local $D_{5 \mathrm{~h}}$ symmetry have been shown to exhibit exceedingly large energy barriers to magnetization reversal exceeding $1000 \mathrm{~cm}^{-1} \cdot{ }^{[25-27]}$ Furthermore, engendering metal ion-radical pairs has proven successful to substantially increase the coercivity and operation temperatures of magnetic materials. ${ }^{[28-32]}$ Alternating current (ac)

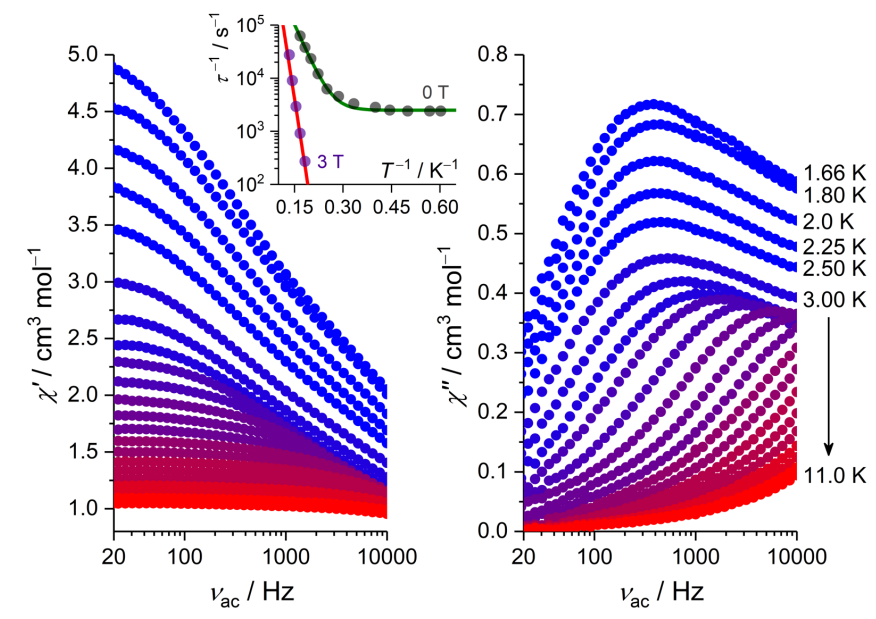

Figure 5. In-phase ( $\chi^{\prime}$, left) and out-of-phase ( $\chi^{\prime \prime}$, right) ac susceptibility data for polycrystalline Dy obtained at selected temperatures and in the absence of a static (dc) magnetic field. Inset: temperature dependence of the paramagnetic relaxation rate, $\tau^{-1}$, vs the reciprocal temperature. The solid lines are simulations as described in the main text. 
susceptibility measurements of Dy reveal the existence of slow relaxation of magnetization in the absence of a dc magnetic field (Fig. 5). The temperature of the spin-lattice relaxation rate, approximated from the maxima of the $\chi^{\prime \prime}\left(v_{\mathrm{ac}}\right)$ as $\tau^{-1}=2 \pi v_{\mathrm{ac}}$ (Fig. 5), was modelled a sum of a term governing the temperature independent quantum tunneling of the magnetization (QTM) and a term representing the two-phonon Orbach process, that is often invoked to rationalize the relaxation in lanthanide-based singlemolecule magnets:

$$
\tau(T)^{-1}=\tau_{\mathrm{QTM}}{ }^{-1}+\tau_{0}^{-1} \mathrm{e}^{-\Delta / k_{\mathrm{B}} T}
$$

The $\tau$ vs $T$ data are well described by $\tau_{\mathrm{QTM}}=0.4 \mu$ s and $\Delta / h c=$ $22 \mathrm{~cm}^{-1}\left(\tau_{0}^{-1}=1.2 \times 10^{-7} \mathrm{~s}\right.$; Fig. 5, green trace). This value of $\Delta$ is dwarfed by those of other $D_{5 \mathrm{~h}}$ symmetric SMMs, however, these systems all display the presence of strongly ligand-field perturbing ligands in the axial positions, which contrasts the weakly coordinating iodide ligands in $\mathbf{D y}$. The application of a sizable, static dc magnetic field of $3 \mathrm{~T}$ breaks the tunneling pathway (Figs S9-11, Supporting Information) and the $\tau$ vs $T$ data can be modelled by the inclusion of the Orbach process only $\left(\Delta / h c=63 \mathrm{~cm}^{-1}\right.$ and $\tau_{0}^{-1}=3.5 \times 10^{-10} \mathrm{~s}$; Fig. 5, red trace $)$.

The isolation of ddQCs and Archimedean tessellations of lanthanide metal-organic networks on metallic surfaces was attributed to significant interactions with the substrate that enforces planarization of the material. ${ }^{[10]}$ Indeed, the equatorial linking of the kinetically labile trans $-\left\{\mathrm{MI}_{2}\right\}^{+}$units may be considered as an analogue of the theoretical scenario of soft, isotropic particles moving in $2 \mathrm{D}$, that has been utilized to explain the formation of ddQCs. ${ }^{[33,34]}$ Herein, we have demonstrated the possibility to construct chemically well-defined Archimedean tessellations of the trivalent lanthanide ions in MOF structures as exemplified by Gd, Gd', and Dy. The tweaking of these systems towards their quasicrystalline variants necessitates the inclusion of local 6-fold ( $D_{6 \mathrm{~h}}$ symmetric) nodes (cf. Fig. 1a), which, despite the large ionic radii, are uncommon for lanthanide complexes. ${ }^{[35]}$ The structure of $\mathbf{G d}^{\prime}$ is remarkable as it gives a prospect on how to introduce the triangle-rich regular triangular motif without the highly uncommon coordination of six ligands in the equatorial plane, necessary to form ddQCs. Whilst previous reports have shown the viability of lanthanide atoms to act as nodes in surface-confined ATs and ddQC, the strategy presented herein constitutes the first generalizable approach to bulk MOF materials possessing both periodic, aperiodic and fractal geometries.

\section{Acknowledgements}

K.S.P. thanks the VILLUM Foundation for a VILLUM Young Investigator grant (15374), the Carlsberg Foundation for a research infrastructure grant (CF-17-0637), the Independent Research Fund Denmark for a Sapere Aude: DFF-Starting Grant, the Danish Society of the Dissemination of Science for a Kirstine Meyer's Memorial Award. H.C., S.K., and K.S.P. thank the Danish National Committee for Research Infrastructure for funding (ESS Lighthouse "SMART").

\section{Conflict of interest}

The authors declare no conflict of interest.

Keywords: lanthanides $\cdot$ single-molecule magnets $\cdot$ metalorganic frameworks $\cdot$ quasicrystal approximants

[1] J. J. Oppenheim, G. Skorupskii, M. Dincă, Chem. Sci. 2020, 11 11094-11103.

[2] K. Deguchi, S. Matsukawa, N. K. Sato, T. Hattori, K. Ishida, H. Takakura, T. Ishimasa, Nat. Mater. 2012, 11, 1013-1016.

[3] Z. V. Vardeny, A. Nahata, A. Agrawal, Nat. Photonics 2013, 7, 177184.

[4] L. Kormoš, P. Procházka, A. O. Makoveev, J. Čechal, Nat. Commun. 2020, 11, 1-7.

[5] X. Zeng, G. Ungar, Y. Liu, V. Percec, A. E. Dulcey, J. K. Hobbs, Nature 2004, 428, 157-160.

[6] S. Lee, M. J. Bluemle, F. S. Bates, Science (80-. ). 2010, 330, 349353.

[7] T. Dotera, J. Polym. Sci. Part B Polym. Phys. 2012, 50, 155-167.

[8] D. Écija, J. I. Urgel, A. C. Papageorgiou, S. Joshi, W. Auwärter, A. P. Seitsonen, S. Klyatskaya, M. Ruben, S. Fischer, S. Vijayaraghavan, et al., Proc. Natl. Acad. Sci. U. S. A. 2013, 110, 6678-6681.

[9] J. I. Urgel, D. Ecija, W. Auwärter, A. C. Papageorgiou, A. P. Seitsonen, S. Vijayaraghavan, S. Joshi, S. Fischer, J. Reichert, J. V. Barth, J. Phys. Chem. C 2014, 118, 12908-12915.

[10] J. I. Urgel, D. Écija, G. Lyu, R. Zhang, C. A. Palma, W. Auwärter, N. Lin, J. V. Barth, Nat. Chem. 2016, 8, 657-662.

[11] V. Smetana, S. P. Kelley, A. Mudring, R. D. Rogers, Sci. Adv. 2020, 6 , eaay 7685 .

[12] L. Voigt, M. Kubus, K. S. Pedersen, Nat. Commun. 2020, 11, 1-6.

[13] A. Carrington, J. dos Santos-Veiga, Mol. Phys. 1962, 5, 21-29.

[14] M. S. Denning, M. Irwin, J. M. Goicoechea, Inorg. Chem. 2008, 47, 6118-6120.

[15] K. Izod, S. T. Liddle, W. Clegg, Inorg. Chem. 2004, 43, 214-218.

[16] G. Heckmann, M. Niemeyer, J. Am. Chem. Soc. 2000, 122, 42274228.

[17] M. R. Macdonald, J. E. Bates, J. W. Ziller, F. Furche, W. J. Evans, J. Am. Chem. Soc. 2013, 135, 9857-9868.

[18] G. A. Heath, L. J. Yellowlees, P. S. Braterman, Chem. Phys. Lett. 1982, 92, 646-648.

[19] T. N. Hooper, J. Schnack, S. Piligkos, M. Evangelisti, E. K. Brechin, Angew. Chemie - Int. Ed. 2012, 51, 4633-4636.

[20] A. Bencini, D. Gatteschi, Electron Paramagnetic Resonance of Exchange Coupled Systems, Springer Berlin Heidelberg, 1990.

[21] W. H. Press, S. A. Teukolsky, W. T. Vetterling, B. P. Flannery, Numerical Recipes in C: The Art of Scientific Computing, Cambridge University Press, Cambridge, 1992.

[22] J. A. Degayner, I. R. Jeon, T. D. Harris, Chem. Sci. 2015, 6, 66396648.

[23] L. Bogani, C. Sangregorio, R. Sessoli, D. Gatteschi, Angew. Chemie - Int. Ed. 2005, 44, 5817-5821.

[24] K. Berlot, L. Bogani, A. Caneschi, D. Gatteschi, R. Sessoli, J. Am. Chem. Soc. 2006, 128, 7947-7956.

[25] Y. S. Ding, N. F. Chilton, R. E. P. Winpenny, Y. Z. Zheng, Angew. Chemie - Int. Ed. 2016, 55, 16071-16074.

[26] Y. S. Ding, K. X. Yu, D. Reta, F. Ortu, R. E. P. Winpenny, Y. Z. Zheng, N. F. Chilton, Nat. Commun. 2018, 9, 1-10.

[27] K. X. Yu, J. G. C. Kragskow, Y. S. Ding, Y. Q. Zhai, D. Reta, N. F. Chilton, Y. Z. Zheng, Chem 2020, 6, 1777-1793.

[28] S. Demir, M. I. Gonzalez, L. E. Darago, W. J. Evans, J. R. Long, Nat. Commun. 2017, 8, 1-9.

[29] J. D. Rinehart, M. Fang, W. J. Evans, J. R. Long, Nat. Chem. 2011, 3, 538-542.

[30] J. D. Rinehart, M. Fang, W. J. Evans, J. R. Long, J. Am. Chem. Soc. 2011, 133, 14236-14239.

[31] C. A. Gould, L. E. Darago, M. I. Gonzalez, S. Demir, J. R. Long, Angew. Chemie - Int. Ed. 2017, 56, 10103-10107.

[32] P. Perlepe, I. Oyarzabal, A. Mailman, M. Yquel, M. Platunov, I. Dovgaliuk, M. Rouzières, P. Négrier, D. Mondieig, E. A. Suturina, et al., Science (80-. ). 2020, 370, 587-592.

[33] K. Barkan, M. Engel, R. Lifshitz, Phys. Rev. Lett. 2014, 113, 1-5

[34] A. S. Keys, S. C. Glotzer, Phys. Rev. Lett. 2007, 99, 1-4.

[35] A. B. Canaj, S. Dey, E. R. Martí, C. Wilson, G. Rajaraman, M. Murrie, Angew. Chemie 2019, 131, 14284-14289. 